\title{
An Origin of CMR: Competing Phases and Disorder-Induced Insulator-to-Metal Transition in Manganites
}

\author{
Yukitoshi Motome $^{1}$, Nobuo Furukawa ${ }^{2}$, and Naoto Nagaosa ${ }^{345}$ \\ 1 RIKEN (The Institute of Physical and Chemical Research), 2-1 Hirosawa, \\ Saitama 351-0198, Japan motome@riken.jp \\ 2 Department of Physics, Aoyama Gakuin University, 5-10-1 Fuchinobe, \\ Sagamihara, Kanagawa 229-8558, Japan furukawa@phys . aoyama.ac.jp \\ 3 CREST, Department of Applied Physics, University of Tokyo, 7-3-1 Hongo, \\ Bunkyo-ku, Tokyo 113-8656, Japan nagaosa@appi.t.u-tokyo.ac.jp \\ 4 Correlated Electron Research Center, AIST, Tsukuba Central 4, 1-1-1 Higashi, \\ Tsukuba, Ibaraki 305-8562, Japan \\ 5 Tokura Spin SuperStructure Project, ERATO, Japan Science and Technology \\ Corporation, c/o AIST, Tsukuba Central 4, 1-1-1 Higashi, Tsukuba, Ibaraki \\ 305-8562, Japan
}

We theoretically explore the mechanism of the colossal magnetoresistance in manganese oxides by explicitly taking into account the phase competition between the double-exchange ferromagnetism and the charge-ordered insulator. We find that quenched disorder causes a drastic change of the multicritical phase diagram by destroying the charge-ordered state selectively. As a result, there appears a nontrivial phenomenon of the disorder-induced insulator-to-metal transition in the multicritical regime. On the contrary, the disorder induces a highly-insulating state above the transition temperature where charge-ordering fluctuations are much enhanced. The contrasting effects provide an understanding of the mechanism of the colossal magnetoresistance. The obtained scenario is discussed in comparison with other theoretical proposals such as the polaron theory, the Anderson localization, the multicritical-fluctuation scenario, and the percolation scenario.

\section{Introduction}

Colossal magnetoresistance (CMR) in perovskite manganese oxides $A \mathrm{MnO}_{3}$ has attracted much attention in the physics of strongly-correlated electron systems $[1,2,3]$. In these materials, carrier doping by chemical substitutions of $A$-site ions, e.g., Ca substitutions into La-site in $\mathrm{LaMnO}_{3}$, leads to a ferromagnetic metal (FM) at low temperatures. In this regime, the resistivity shows a rapid decrease by applying an external magnetic field near the critical point, which is called the CMR effect. The doped FM state and the negative magnetoresistance are basically understood by the Zener's double-exchange (DE) interaction $[4,5]$. In the Zener's scenario, the system consists of con- 
duction electrons and localized spins, and there is a strong ferromagnetic Hund's coupling between them. Through this strong correlation, the external magnetic field which aligns the localized spins ferromagnetically increases the kinetic energy of electrons to induce a metallic state.

Recent development in experiments, partly promoted by potential applications to electronic engineering such as spintronics devices, has achieved to enhance the CMR effect; the resistivity sharply decreases on the order of $10^{4}-10^{6}$ by the magnetic field of only a few Tesla. One of the important features of the enhanced CMR is a characteristic temperature dependence of the resistivity at zero magnetic field. The resistivity shows good metallic behavior below the ferromagnetic transition temperature $T_{\mathrm{C}}$ while it shows highlyinsulating behavior above $T_{\mathrm{C}}$. That is, the resistivity shows a steep increase toward $T_{\mathrm{C}}$ from above and suddenly drops near $T_{\mathrm{C}}$. The highly-insulating state just above $T_{\mathrm{C}}$ is very sensitive to the magnetic field, which leads to the enhanced CMR effect. Therefore, the origin of the highly-insulating state above $T_{\mathrm{C}}$ is a key to understand the mechanism of the enhanced CMR effect.

Either the highly-insulating nature or the huge response to the external magnetic field cannot be explained by the simple DE theory. There have been many theoretical proposals which attempt to explain them. Several scenarios are based on the single-particle picture such as spin polaron [6] or Jahn-Teller (JT) polaron theory [7, 8] and Anderson localization scenario by quenched disorder $[9,10]$. Recently, some attempts have also been made to understand the enhanced CMR as a cooperative phenomenon due to the many-body correlation, such as the multicritical-fluctuation scenario [11] and the percolation scenario $[12,13]$. It is highly desired to clarify which is a suitable picture.

Several experiments indicate that the importance of a keen competition between different phases and quenched disorder for the CMR phenomena. One of the systematic investigations has been explored in a new class of materials $A_{1 / 2} \mathrm{Ba}_{1 / 2} \mathrm{MnO}_{3}[14,15,16]$. It is found that under a special condition of the synthesis, $A$ ions and $\mathrm{Ba}$ ions constitute a periodic layered structure. In these $A$-site ordered materials, every $\mathrm{Mn}$ site has an equivalent environment of the surrounding ions, and therefore, there is no disorder from the alloying effect. In a usual synthesis process or by annealing the $A$-site ordered materials, one obtains the materials in which $A$ and $\mathrm{Ba}$ ions distribute randomly, and there is structural and electrostatic disorder at Mn sites. Since the systematic change of the average radius of $A$-site ions is known to modify the electron bandwidth, it is now possible to control both electron correlation and quenched disorder in a systematic way in these new materials.

Figure 1 shows the phase diagram of this class of materials obtained by Akahoshi et al. [16]. Open symbols connected by the solid line in the figure are the results in the $A$-site ordered materials. There, the system shows a typical multicritical phase diagram, that is, the FM transition temperature $T_{\mathrm{C}}$ and the charge-ordered insulator (COI) transition temperature $T_{\mathrm{CO}}$ meet 


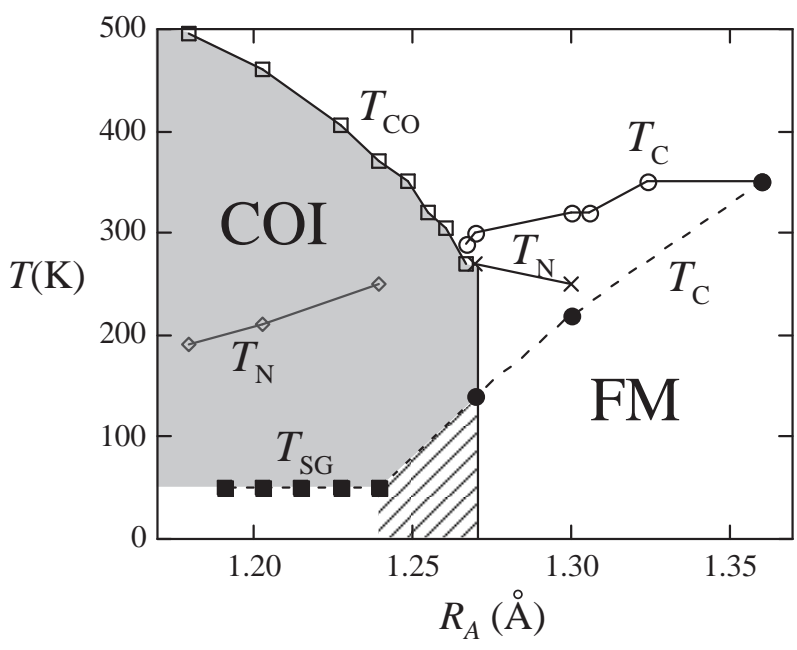

Fig. 1. Experimental phase diagram for $A_{1 / 2} \mathrm{Ba}_{1 / 2} \mathrm{MnO}_{3}$ obtained in Ref. [16]. The horizontal axis is the averaged ionic radius of the $A$-site ion. $T_{\mathrm{C}}, T_{\mathrm{CO}}, T_{\mathrm{N}}$, and $T_{\mathrm{SG}}$ denote the transition temperatures for FM, COI, the antiferromagnetic state, and the glassy state, respectively. The hatched area is the disorder-induced COI-to-FM transition regime. The shaded area shows the region where COI is destroyed by introducing the $A$-site disorder. See the text and Ref. [16] for details.

with each other at almost the same temperature (the multicritical point). In the disordered materials, the multicritical phase diagram shows a drastic change in an asymmetric manner as shown by the closed symbols connected by the dashed line in the figure. That is, the FM state is robust in spite of a suppression of $T_{\mathrm{C}}$, whereas the COI state completely disappears and is replaced by a glassy state at very low temperatures below $T_{\mathrm{SG}}$. The multicritical point is shifted to the left side of the phase diagram, and at the same time, is suppressed down to a lower temperature. Consequently, there appears a nontrivial regime in which the disorder induces the transition from COI to FM (the hatched area in Fig. 1).

An important observation is that in this regime of the disorder-induced COI-to-FM transition, the resistivity shows the characteristic temperature dependence mentioned above, i.e., the highly-insulating state above $T_{\mathrm{C}}$ followed by a sudden drop below $T_{\mathrm{C}}$. There, the typical enhanced CMR effect is obtained by applying a small external magnetic field [16]. Similar phenomena are also observed in $A_{0.55}(\mathrm{Ca}, \mathrm{Sr})_{0.45} \mathrm{MnO}_{3}[17,18]$ and in $\mathrm{Cr}$ doping in $A_{1 / 2} \mathrm{Ca}_{1 / 2}(\mathrm{Mn}, \mathrm{Cr}) \mathrm{O}_{3}[19,20,21,22]$. Another crucial observation is that strong fluctuations of charge and lattice orderings are observed in the highlyinsulating state above $T_{\mathrm{C}}$, but they are largely suppressed below $T_{\mathrm{C}}$ [18]. This reentrant behavior appears to correlate with the temperature dependence of the resistivity. These experimental results strongly suggest that the disorder 
plays a significant role in the multicritical phenomena to induce the enhanced CMR effect.

The purpose of this Contribution is to investigate the phase competition in the multicritical regime and the disorder effect theoretically. We will explore a comprehensive understanding of the enhanced CMR effect on the basis of a cooperative-phenomenon picture rather than a single-particle one. In the next section 2, we introduce an extended DE model to describe the phase competition, and briefly explain the numerical method. The results are presented in Sec. 3. We discuss the origin of CMR on the basis of our results in Sec. 4. We also compare our results with experimental data and other theoretical proposals. Sec. 5 is devoted to summary and concluding remarks.

\section{Model and Method}

In the present study, we consider a minimal model which captures the competition between FM and COI. We take account of the conventional DE interaction as a stabilization mechanism of FM state. As for COI, we consider one of the simplest mechanisms, i.e., the electron-phonon coupling to the breathing-type distortions [23]. The explicit form of the Hamiltonian reads $[24]$

$$
\begin{aligned}
H= & -t \sum_{\langle i j\rangle \sigma}\left(c_{i \sigma}^{\dagger} c_{j \sigma}+\text { H.c. }\right)-J_{\mathrm{H}} \sum_{i} \sigma_{i}^{z} S_{i} \\
& -g \sum_{i} n_{i} Q_{i}+\frac{1}{2} \sum_{i} Q_{i}^{2}+\frac{\lambda}{2} \sum_{\langle i j\rangle} Q_{i} Q_{j}+\sum_{i} \epsilon_{i} n_{i},
\end{aligned}
$$

where the summation with $\langle i j\rangle$ is taken over the nearest-neighbor sites $i$ and $j$, and the index $\sigma$ denotes the spin of conduction electrons. The first line of Eq. (1) is for the DE part; the first term describes the kinetic energy of the single-band conduction electrons with the transfer integral $t$, and the second term denotes the ferromagnetic Hund's coupling between the conduction electron spin $\sigma_{i}$ and the localized spin $S_{i}$. For simplicity, we consider the limit of $J_{\mathrm{H}} \rightarrow \infty$ and the coupling of the Ising symmetry, i.e., $S_{i}= \pm 1$, which retains the essential physics of the DE ferromagnetism [25]. The first term in the second line of Eq. (1) describes the electron-phonon coupling of

the breathing type where $g$ is the coupling constant, $n_{i}=\sum_{\sigma} c_{i \sigma}^{\dagger} c_{i \sigma}$ is the local electron density, and $Q_{i}$ is the amplitude of the distortion at the site $i$. The next two terms denote the elastic energy of distortions. The latter term describes a cooperative aspect of the lattice distortion, where $\lambda$ is taken to be positive because a shrinkage (expansion) of a $\mathrm{MnO}_{6}$ octahedron tends to expand (shrink) the neighboring $\mathrm{MnO}_{6}$ octahedra. Lattice distortions are treated as classical objects for simplicity. The last term in Eq. (1) is for the quenched disorder which couples to conduction electrons. In real materials, 
the alloying effect of $A$-site ions in $A \mathrm{MnO}_{3}$ as well as of the Cr substitution into the Mn sites causes structural and electrostatic disorder, which modifies the on-site potential through the Madelung energy. Here, we mimic this by the random on-site energy $\epsilon_{i}$.

We consider the model (1) on the two-dimensional (2D) square lattice in the half-doped case, i.e., 0.5 electron per site on average. We set the halfbandwidth in the case of $J_{\mathrm{H}}=g=\epsilon_{i}=0$ as an energy unit, i.e., $W=4 t=1$. We take $\lambda=0.1$ hereafter. We consider the binary-type distribution of the random potential, i.e., $\epsilon_{i}= \pm \Delta$.

When $g$ is small (large bandwidth), the DE part is dominant and stabilizes FM at low temperatures. On the other hand, when $g$ is large (small bandwidth), the electron-phonon coupling becomes dominant and induces the checkerboard-type charge order (CO) with the wave number $(\pi, \pi)$. Hence, the competition between FM and COI is expected in our model (1) by controlling $g / W$. In the $A$-site ordered materials, the $A$-site substitution modifies mainly the bandwidth of conduction electrons through changes of the length and the angle of Mn-O-Mn bonds. The right (left) hand side of the phase diagram in Fig. 1 corresponds to larger (smaller) bandwidth regime where FM (COI) is stabilized at low temperatures. In the $A$-site disordered materials, the situation is more complicated; the $A$-site substitution may change the bandwidth as well as the strength of the disorder. In the following, we investigate the model (1) by changing both $g$ and $\Delta$ systematically to understand the physics of the competing phases observed in experiments.

We study thermodynamic properties of the model (1) by employing the Monte Carlo (MC) simulation. In the MC sampling, physical quantities are averaged for configurations of localized spins $\left\{S_{i}\right\}$ and lattice distortions $\left\{Q_{i}\right\}$ which are randomly generated by using the importance sampling technique. The MC weight is calculated by the diagonalization of the electronic part for a given configuration of $\left\{S_{i}\right\}$ and $\left\{Q_{i}\right\}$. In the presence of disorder, we take a quenched random average for different configurations of the on-site random potential $\left\{\epsilon_{i}\right\}$. For the details of MC calculations, readers are referred to Ref. [24]. In the present system, since the competition between different orders as well as the spatial inhomogeneity due to the disorder is important, it is crucial to distinguish short-range correlations and long-range orders by applying the systematic finite-size scaling analyses [26].

\section{Results}

\subsection{Disorder Effect on Multicritical Phase Diagram}

In the pure case without disorder $(\Delta=0)$, the model (1) shows the multicritical phase diagram as shown in Fig. 2 (a). There, we have four different phases; the high-temperature para phase, FM phase in the small $g / W$ regime, 
COI phase in the large $g / W$ regime, and the coexisting phase of ferromagnetism and charge ordering $(\mathrm{F}+\mathrm{COI})$ in between. Note that the coexisting $\mathrm{F}+\mathrm{COI}$ state is uniform and not phase-separated. Thus, the phase diagram shows a tetracritical topology [27]. From the systematic study of the density of states (not shown here), we find a metal-insulator transition at the phase boundary between FM and F+COI phases.

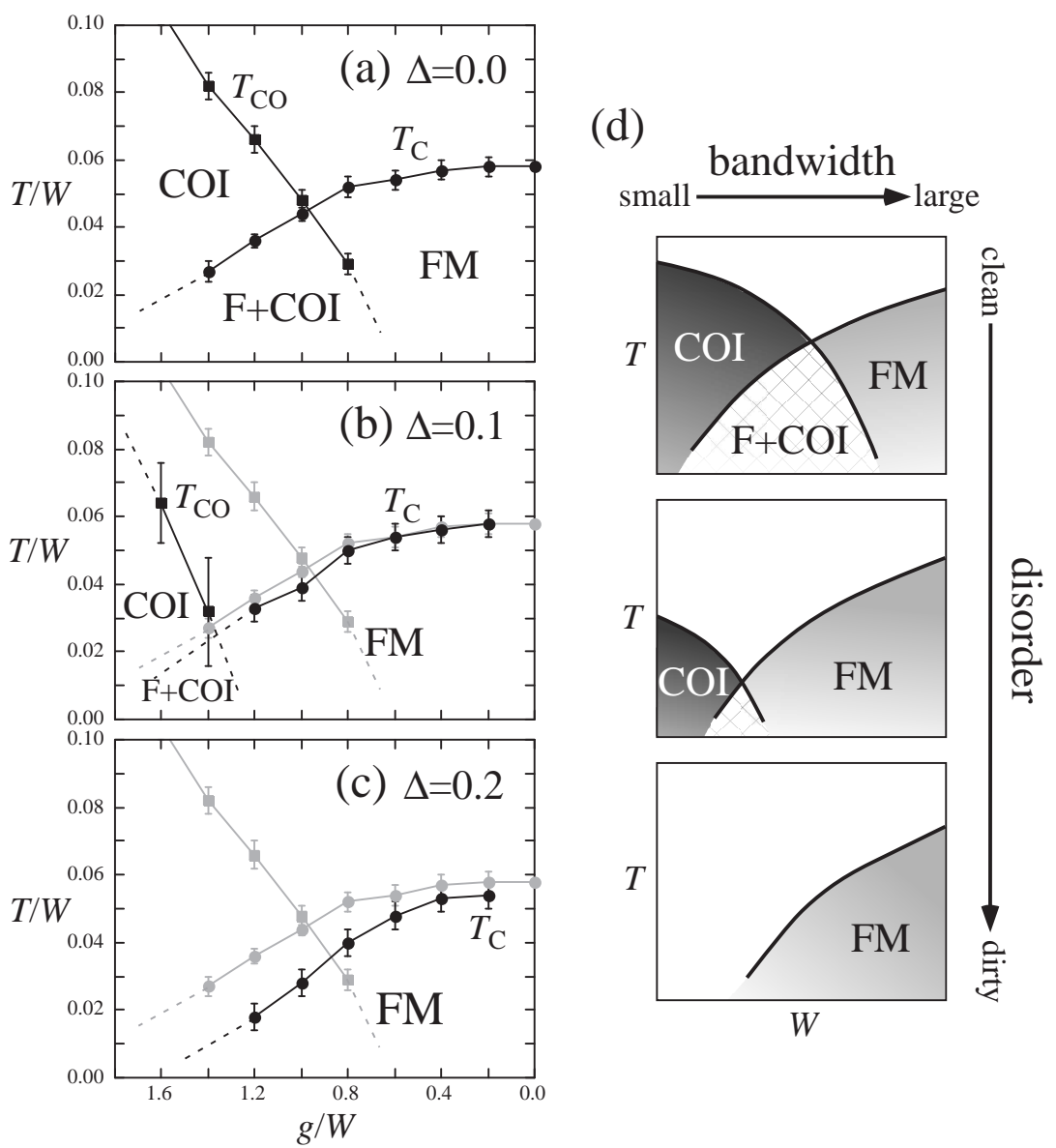

Fig. 2. (a)-(c) Phase diagrams of the model (1) for the change of the disorder strength $\Delta$. Circles (squares) denote the transition temperatures for the ferromagnetism (the charge ordering) estimated by using the finite-size scaling up to $8 \times 8$ sites. The lines are guides for the eyes. In (b) and (c), the results for the pure case (a) are shown by gray symbols and lines for comparison. The change of the phase diagram is schematically summarized in $(d)$. 
When we introduce the disorder to the system, the multicritical phase diagram shows the systematic and drastic change as shown in Figs. 2 (b) and (c). The FM phase is robust to the disorder showing a slight decrease of $T_{\mathrm{C}}$, whereas the COI phase is surprisingly unstable to the disorder. The critical temperature $T_{\mathrm{CO}}$ rapidly decreases with increasing the strength of disorder $\Delta$, and the COI phase finally collapses at $\Delta \sim 0.2 W$ in the parameter range of the present MC calculations.

The asymmetric change of the multicritical phase diagram for the disorder is schematically summarized in Fig. 2 (d). In the following, we will explore the mechanism of this change to clarify the origin of the enhanced CMR.

\subsection{Fragility of Commensurate Insulator against Disorder}

FM state is robust to the disorder because the DE ferromagnetism is stabilized by the kinetics of conduction electrons. $T_{\mathrm{C}}$ is proportional to the kinetic energy which gradually decreases with the disorder [28]. On the contrary, we found that $\mathrm{COI}$ is surprisingly fragile against the disorder. The fragility is understood by the out-of-phase pinning phenomenon as follows.

Figure 3 (a) shows the MC results of the density of states (DOS) in the COI regime. Even when the disorder destroys the long-range CO, the gap structure in DOS remains robust as shown in the figure. This means that short-range correlations survive and local lattice distortions persist to open the gap. Therefore, the fragility of the COI phase is not due to the rapid decrease of the amplitude of lattice distortions, but due to the disturbance of the phase of the commensurate ordering. The random potential to electrons is 'a random field' to $\mathrm{CO}$, and it easily pins the commensurate ordering pattern with introducing antiphase domain walls as schematically shown in Fig. 3 (b).

\subsection{Highly-Insulating State above $T_{\mathrm{C}}$}

The out-of-phase pinning picture suggests that there remain short-range charge correlations and fluctuations in the region where the long-range CO is destroyed by the disorder. This is indeed the case. Figure 4 shows the temperature dependences of the susceptibility of the staggered lattice distortion which is calculated by fluctuations of the COI order parameter. Although the long-range $\mathrm{CO}$ is destroyed by the disorder and the diverging behavior at $T_{\mathrm{CO}}$ in the pure case is smeared out, fluctuations of charge and lattice orderings remain finite and are enhanced toward $T_{\mathrm{C}}$ even at a finite $\Delta$. This could be regarded as a reminiscence of the multicritical phenomenon in the pure case [11].

We note that the fluctuations are suppressed for large values of $\Delta$. We consider that for very strong disorder, the system may favor rather a static state with small COI clusters rather than the fluctuating state. This crossover will be discussed in Sec. 4.3. 


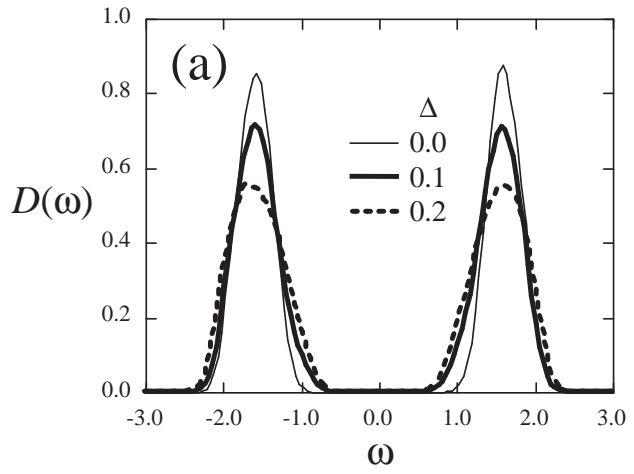

(b)

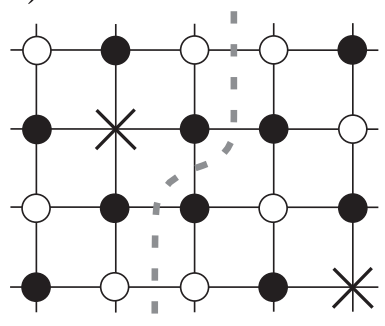

Fig. 3. (a) The density of states at $g=1.6$ and $T=0.016$ calculated for $8 \times 8$-sites systems. (b) A schematic picture of the out-of-phase pinning for the checkerboardtype charge ordering. White (black) circles denote electrons (holes), and crosses are the random pinning centers. The dashed gray curve shows an antiphase domain wall.

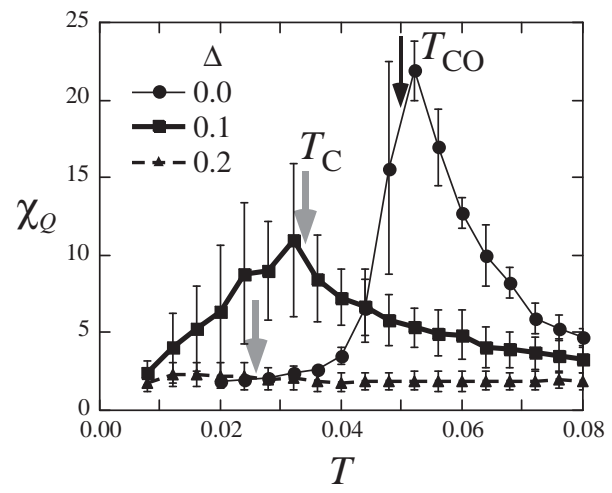

Fig. 4. Temperature dependence of the staggered susceptibility of the lattice distortion calculated at $g=1.0$ for $8 \times 8$-sites systems. The gray arrows indicate the values of $T_{\mathrm{C}}$ in the presence of disorder.

The remaining fluctuations of $\mathrm{CO}$ affect the electronic states in the hightemperature regime. Figures 5 show the MC results of (a) DOS and (b) the optical conductivity in this regime. As $\Delta$ becomes larger, the dip in DOS at the Fermi energy becomes deeper, and the low-energy weight of $\sigma(\omega)$ becomes smaller to develop a quasi-gap feature. Thus, the disorder induces fluctuations of $\mathrm{CO}$, and tends to make the system insulating in the hightemperature regime. 

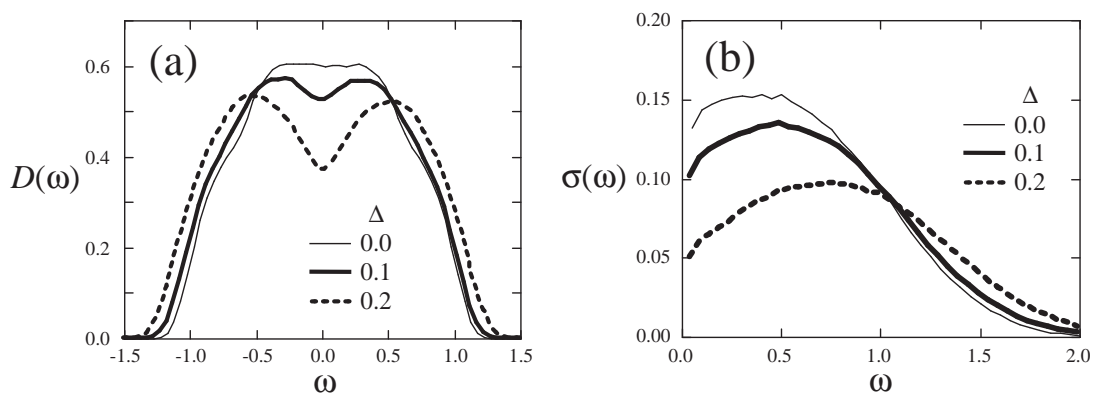

Fig. 5. (a) The density of states $(8 \times 8$ sites $)$ and (b) the optical conductivity $(6 \times 6$ sites) at $g=0.8$ and $T=0.06$.
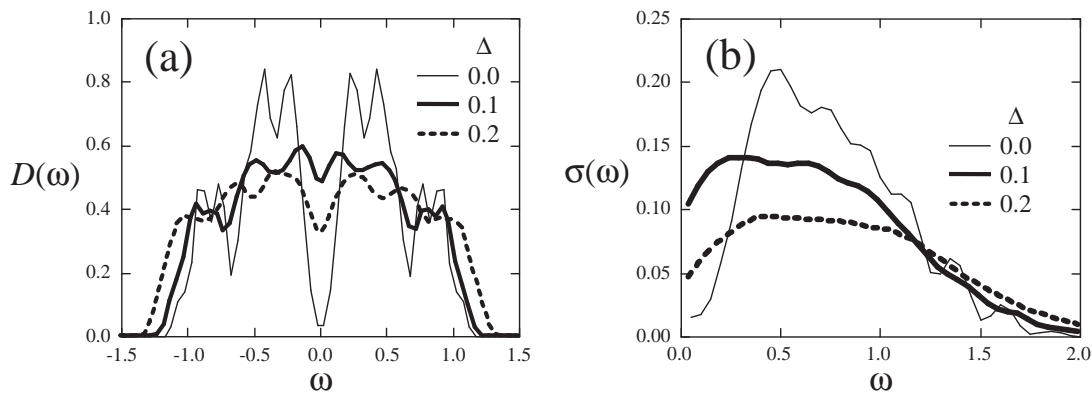

Fig. 6. (a) The density of states at $g=0.8$ and $T=0.016(8 \times 8$ sites $)$. (b) The optical conductivity at $g=0.8$ and $T=0.02(6 \times 6$ sites $)$.

\subsection{Disorder-Induced Insulator-to-Metal Transition}

In contrast to the enhanced insulating nature at high temperatures, we find a remarkable phenomenon at low temperatures in the multicritical regime, that is, the disorder-induced insulator-to-metal transition. Figures 6 show (a) DOS and (b) the optical conductivity close to the metal-insulator phase boundary. In the pure case $(\Delta=0)$, DOS shows a finite gap and $\sigma(\omega)$ has a gap structure due to the long-range CO. When we introduce the disorder, the gap is suddenly filled and there appears a finite DOS at the Fermi energy. At the same time, the low-energy spectral weight of $\sigma(\omega)$ rapidly increases. These strongly indicate that the disorder induces the transition from the commensurate insulator to a metallic state. Such a phenomenon caused by the multicritical phase competitions is surprising and counter-intuitive because in general the disorder tends to localize electrons and to make the system more insulating.

We note that the optical conductivity in the multicritical regime in Fig. 6 (b) as well as in Fig. 5 (b) shows drastic reconstruction in the wide energy range up to $\omega \sim W$ by a small disorder of only the order of $0.1 W$. This is a consequence of the cooperative phenomena in the phase-competing regime. 


\subsection{Brief Summary of Results}

Here, we give a short summary of our results. MC simulation revealed that our model (1) exhibits the multicritical phenomenon due to the competition between FM and COI states, and the competing phases show the asymmetric response to the disorder. The COI phase is very unstable to the disorder while the FM phase is rather robust, which leads to the disorder-induced insulator-to-metal transition at low temperatures. In spite that the longrange $\mathrm{CO}$ is destroyed at low temperatures, the short-range correlations of $\mathrm{CO}$ are enhanced by the disorder at high temperatures, which tends to make the system more insulating. Thus, we obtain reentrant behavior, i.e., charge and lattice correlations are enhanced as decreasing temperature, but they are suppressed below $T_{\mathrm{C}}$. This is considered to be entropy-driven reentrant behavior; the short-range $\mathrm{CO}$ state has rather high entropy related to configurations of the antiphase domain walls, and is favored at high temperatures

due to the high entropy. We consider that this reentrant behavior is an origin of CMR phenomena as discussed in Sec. 4.1.

Our results elucidate an important role of the contrasting nature of the competing phases. The FM phase is stabilized by the kinetics of conduction electrons, and has a long-range ordering with the wave number $(0,0)$. On the other hand, the COI phase is stabilized by a commensurate lattice ordering with the wave number $(\pi, \pi)$. The former is robust but the latter is fragile against the out-of-phase pinning by disorder. The competition between the uniform metal and the commensurate insulator plays a central role.

\section{Discussions}

\subsection{Origin of CMR}

In this section, we discuss the origin of CMR suggested by our results. We found in Sec. 3 the contrasting effects of disorder on the electronic states in the phase competing regime, i.e., the insulator-to-metal transition at low temperatures and the enhanced insulating nature at high temperatures. The former occurs in the hatched area in the schematic phase diagram shown in Fig. 7 (a). The latter is conspicuous in the shaded area in Fig. 7 (a) where we found the enhanced fluctuations of charge and lattice orderings.

The contrasting effects of disorder and the resultant reentrant behavior of charge and lattice correlations provide a key to understand the enhanced CMR effect observed in experiments. Schematic temperature dependences of the resistivity along the downward arrow in Fig. 7 (a) are shown in Fig. 7 (b), suggested by our numerical results of $\sigma(\omega)$ in Sec. 3. In the pure case with $\Delta=0$, the resistivity sharply increases below the COI transition temperature $T_{\mathrm{CO}}$ as shown by the dashed curve in Fig. 7 (b). When the disorder 
is introduced into the system, the high-temperature resistivity increases because the disorder enhances the insulating nature there. On the contrary, at a low temperature, we have the insulator-to-metal transition induced by the disorder, where the resistivity should show a sudden drop. Hence, we obtain the characteristic temperature dependence for $\Delta \neq 0$ as shown by the solid curve in Fig. 7 (b). Note that the temperature dependence is in accord with that of charge and lattice fluctuations found in Fig. 4, which illuminates the close relation between the resistivity and these fluctuations.

The enhanced insulating state near above $T_{\mathrm{C}}$ is known to show a huge response to a small external magnetic field, i.e., the enhanced CMR as mentioned in Sec. 1. Such a huge response is expected in the shaded region in Fig. 7 (a) where fluctuations of charge and lattice orderings are enhanced by the disorder. Thus, the enhanced CMR phenomena can be understood by the contrasting effects of the disorder in the phase competition between the uniform metal and the commensurate insulator.
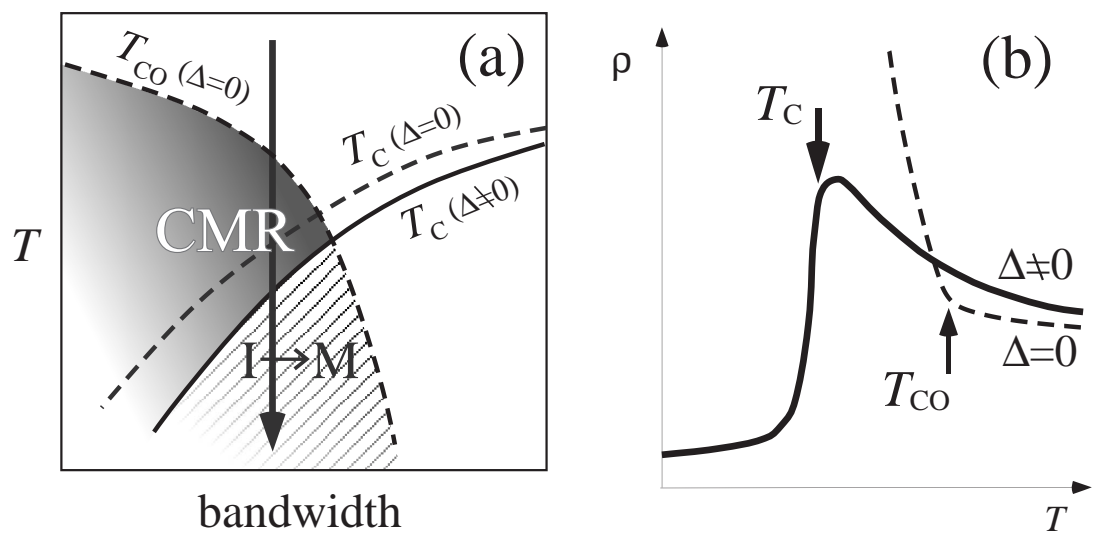

Fig. 7. (a) Schematic phase diagram of the model (1). Dashed curves denote $T_{\mathrm{C}}$ and $T_{\mathrm{CO}}$ in the absence of disorder, and the solid curve denotes $T_{\mathrm{C}}$ in the presence of disorder. See Fig. 2 (d). The hatched area shows the region where the disorderinduced insulator-to-metal transition takes place. The shaded area denotes the relevant regime to the huge CMR effect where COI fluctuations are substantial and the highly-insulating state is realized. (b) Temperature dependences of the resistivity along the downward arrow in (a) suggested by the present study. Dashed and solid curves correspond to the cases without and with disorder, respectively.

\subsection{Comparison with Experimental Results}

Our results well reproduce the experimental results in CMR manganites of the $A$-site ordered/disordered materials $[15,16], A_{0.55}(\mathrm{Ca}, \mathrm{Sr})_{0.45} \mathrm{MnO}_{3}[17,18]$, 
and Cr-doped materials $A_{1 / 2} \mathrm{Ca}_{1 / 2}(\mathrm{Mn}, \mathrm{Cr}) \mathrm{O}_{3}[19,20,21,22]$ in the following aspects;

- multicritical phase diagram in clean cases,

- asymmetric change of the multicritical phase diagram by introducing disorder, in particular, the fragility of COI phase,

- disorder-induced insulator-to-metal transition in the multicritical regime,

- highly-insulating state above $T_{\mathrm{C}}$ in the presence of disorder,

- remaining short-range correlations or fluctuations of charge and lattice orderings in the insulating state above $T_{\mathrm{C}}$ observed in Raman scattering and diffuse $\mathrm{X}$-ray scattering experiments,

- reentrant temperature dependence commonly observed in the resistivity and the charge and lattice fluctuations.

The agreement strongly indicates that our model (1) captures the essential physics of phase competing phenomena and disorder effects on them in CMR materials. We also note that our results give an understanding of the close relation between the charge fluctuations and CMR found in $\mathrm{La}_{1-x} \mathrm{Ca}_{x} \mathrm{MnO}_{3}$ at $x \sim 1 / 3[29,30]$.

\subsection{Comparison with Other Theoretical Scenarios}

Finally, we compare our scenario with other theoretical proposals for the mechanism of CMR. As discussed in Sec. 1, CMR is a kind of localizationdelocalization phenomenon at the ferromagnetic transition temperature $T_{\mathrm{C}}$. The low-temperature metallic phase is basically understood by the Zener's DE mechanism, and hence, the problem is how to understand the hightemperature insulating state above $T_{\mathrm{C}}$.

Several proposals are based on the single-particle picture. One is the polaron scenario including both spin polaron [6] and JT polaron theories $[7,8]$. In this scenario, the high-temperature insulating state is described by the self trapping of electrons to form small polarons. Another scenario is the Anderson localization due to the quenched disorder $[9,10]$. For both scenarios, it is difficult to answer the following questions on the general aspects of the enhanced CMR comprehensively:

- Why does the localized state at high temperatures become unstable at lower temperatures?

- Why does the localization-delocalization transition always coincide with the ferromagnetic transition without fine tuning of parameters?

- Why does the disorder cause the insulator-to-metal transition as observed in the $A$-site ordered manganites?

- Why is CMR observed in wide region of the phase diagram, or why does the localization occur even at highly carrier-doped region?

Hence, the single-particle pictures appear to be insufficient to explain the enhanced CMR. In particular, they cannot explain the experimental fact that 
the 'clean' system shows the multicritical phase diagram while the disorder induces the CMR phenomenon in the multicritical regime.

On the contrary, our results based on the cooperative-phenomenon picture give a comprehensive understanding of CMR as a localization-delocalization phenomenon. Essential ingredients are the phase competition and the disorder, which are found in general in manganites and do not need fine tuning of parameters. Our theory answers why the delocalization occurs at $T_{\mathrm{C}}$ as well as why the disorder induces the insulator-to-metal transition.

We note that there have been proposed other cooperative-phenomenon scenarios. One is the multicritical-fluctuation scenario [11] in which enhanced fluctuations near the multicritical point play an important role in the insulating state above $T_{\mathrm{C}}$. The scaling law for the magnetization curve (the so-called Arrot plot) shows an evidence for this scenario. Although the scaling holds for a class of materials with weak disorder in which the multicritical point is at rather high temperature, it does not show good agreement with the others where the multicritical point is suppressed down to low temperatures by the disorder and the typical CMR is observed.

Another proposal is the percolation scenario which explicitly takes account of the disorder. In this scenario, it is supposed that the disorder induces the percolated mixtures of FM and COI islands below $T^{*}$ which is the transition temperature $T_{\mathrm{C}}$ or $T_{\mathrm{CO}}$ in the absence of the disorder $[12,13]$. The resistivity is determined by the percolating path, and is sensitive to the pattern of the coexisting FM and COI regions which is easily changed by an external magnetic field. However, the theory is limited to a phenomenological level and does not give a microscopic explanation for the disorder-induced insulator-to-metal transition as well as the reentrant behavior of CO fluctuations. Moreover, this rather static picture appears to be incompatible with recent experimental results which indicate a dynamically-fluctuating state just above $T_{\mathrm{C}}[31]$.

Compared with above two scenarios based on the cooperative-phenomenon picture, our theory is considered to interpolate these two limiting pictures, i.e., it elucidates what happens in weak or moderate disorder regime in the phase-competing system. There, short-range correlations or dynamical fluctuations of charge and orbital orderings remain substantial and are relevant to the localization at high temperatures. In our numerical results in Sec. 3, we do not find any clear indication of the percolated cluster formation below $T^{*}[24]$, and such static phase separation may be relevant in rather strong disorder regime where $\mathrm{CO}$ fluctuations are largely suppressed as implied by Fig. 4. Out theory, which describes the crossover from the clean to dirty limits, strongly suggests that the enhanced CMR occurs in the weak or moderate disorder regime with large fluctuations. Note that the unbiased numerical calculations play key roles to reveal the highly-nontrivial properties in the weak or moderate disorder regime . 


\section{Summary and Concluding Remarks}

In this Contribution, we discussed the phase competition between ferromagnetic metal and charge-ordered insulator and the role of the disorder by applying the Monte Carlo calculations to an extended double-exchange model. Highly nontrivial phenomena are revealed such as the disorderinduced insulator-to-metal transition as well as the entropy-driven reentrant behavior of charge-ordering fluctuations. Our results show good agreement with recent experimental results in CMR manganites, and give a comprehensive understanding of the origin of the enhanced CMR effect.

There still remain many open problems. One is the role of other interactions which are neglected in our simplified model. One of the neglected elements is the orbital degree of freedom in the twofold $e_{g}$ orbitals which strongly couples with the JT lattice distortion. This is necessary, at least, to describe a complicated orbital ordering in the COI phase [32], and possibly introduces orbital fluctuations and enhances the insulating nature in the CMR regime as the fluctuations of charge and lattice orderings. Another omitted element is the AF superexchange interaction between localized spins. This is important to reproduce the complicated magnetic structure in the socalled CE phase as well as the A-type AF metallic phase, and possibly plays a substantial role in the glassy state at low temperatures. Recent theoretical study including these elements reported similar results to ours [33] although the numerical analysis is laborious and very limited for such a complicated model thus far.

Another issue is the calculation in realistic 3D systems. The present calculations have been performed in $2 \mathrm{D}$. The pinning effect due to the disorder is sensitive to the dimensionality, which should be carefully examined further. Moreover, in real materials, there may be some spatial correlation between the disorders, in other words, a long-range nature of the influence of disorder through the lattice strain effect or the cooperative effects of lattice distortions [34]. There, the orbital degree of freedom should play an important role through the Jahn-Teller coupling. It is highly desired to examine such effects for realistic electronic models in the higher dimension.

The direct calculation of the resistivity also remains as a hard task for numerical studies. There are several reports on the resistivity in small-size clusters which strongly indicate a huge response to the magnetic field $[23,35]$. To explain the enhanced CMR effect more quantitatively, further investigations are necessary including the development of theoretical tools to calculate the resistivity directly in larger-scale systems.

\section{Acknowledgment}

The authors acknowledge Y. Tokura, Y. Tomioka, and E. Dagotto for fruitful discussions. This work is supported by Grants-in-Aid for Scientific Research 
and NAREGI Nanoscience Project from the Ministry of Education, Culture, Sports, Science, and Technology.

\section{References}

1. A.P. Ramirez, J. Phys: Cond. Matt. 9, 8171 (1997), and references therein.

2. Y. Tokura and N. Nagaosa, Science 288, 462 (2000), and references therein.

3. E. Dagotto, T. Hotta, and A. Moreo, Phys. Rep. 344, 1 (2001), and references therein.

4. C. Zener, Phys. Rev. 82, 403 (1951).

5. N. Furukawa, in Physics of Manganites, edited by T. Kaplan and S. Mahanti (Plenum, New York, 1999), and references therein.

6. C.M. Varma, Phys. Rev. B 54, 7328 (1996).

7. A.J. Millis, P.B. Littlewood, and B.I. Shraiman, Phys. Rev. Lett. 74, 5144 (1995); A.J. Millis, R. Mueller, and B.I. Shraiman, Phys. Rev. B 54, 5405 (1996).

8. H. Röder, J. Zang, and A.R. Bishop, Phys. Rev. Lett. 76, 1356 (1996); J. Zang, A.R. Bishop, and H. Röder, Phys. Rev. B 53, R8840 (1996).

9. B.M. Letfulov and J.K. Freericks, Phys. Rev. B 64, 174409 (2001).

10. E.E. Narimanov and C.M. Varma, Phys. Rev. B 65, 024429 (2002).

11. S. Murakami and N. Nagaosa, Phys. Rev. Lett. 90, 197201 (2003).

12. A. Moreo, S. Yunoki, and E. Dagotto, Science 283, 2034 (1999).

13. J. Burgy et al., Phys. Rev. Lett. 87, 277202 (2001).

14. F. Millange et al., Chem. Mater. 10, 1974 (1998).

15. T. Nakajima et al., J. Phys. Soc. Jpn. 71, 2843 (2002).

16. D. Akahoshi et al., Phys. Rev. Lett. 90, 177203 (2003).

17. Y. Tomioka and Y. Tokura, Phys. Rev. B 66, 104416 (2002).

18. Y. Tomioka et al., Phys. Rev. B 68, 094417 (2003).

19. A. Barnabé et al., Appl. Phys. Lett. 71, 3907 (1997); B. Raveau, A. Maignan, and C. Martin, J. Solid State Chem. 130, 162 (1997).

20. T. Kimura et al., Phys. Rev. Lett. 83, 3940 (1999).

21. Y. Moritomo et al., Phys. Rev. B 60, 9220 (1999).

22. T. Katsufuji et al., J. Phys. Soc. Jpn. 68, 1090 (1999).

23. J.A. Vergés, V. Martín-Mayor, and L. Brey, Phys. Rev. Lett. 88, 136401 (2002).

24. Y. Motome, N. Furukawa, and N. Nagaosa, Phys. Rev. Lett. 91, 167204 (2003).

25. Y. Motome and N. Furukawa, J. Phys. Soc. Jpn. 70, 1487 (2001); 70, 2802 (2001). The Ising symmetry enables us to study finite-temperature phase transitions in $2 \mathrm{D}$ systems.

26. Y. Motome, N. Furukawa, and N. Nagaosa, J. Mag. Mag. Mater., in press.

27. The tetracritical phase diagram can be turned into the bicritical one by explicitly including a competing term between FM and CO, which makes the coexisting F+COI state unstable [26]. The effects of disorder are qualitatively common to both tetracritical and bicritical cases, and therefore we focus on the tetracritical case in this Contribution.

28. Y. Motome and N. Furukawa, Phys. Rev. B 68, 144432 (2003).

29. P. Dai et al., Phys. Rev. Lett. 85, 2553 (2000).

30. C.P. Adams et al., Phys. Rev. Lett. 85, 3954 (2000). 
31. S. Mori, private communications.

32. S. Yunoki, A. Moreo, and E. Dagotto, Phys. Rev. Lett. 81, 5612 (1998).

33. H. Aliaga et al., Phys. Rev. B 68, 104405 (2003).

34. J. Burgy, A. Moreo, and E. Dagotto, Phys. Rev. Lett. 92, 097202 (2004).

35. C. Sen, G. Alvarez, and E. Dagotto, preprint (cond-mat/0401619). 